\title{
Predictive Value of Caprini Venous Thromboembolism Risk Assessment Model for Deep Vein Thrombosis in Intensive Care Unit Non-surgical Patients
}

\author{
Xin Zhang, Wanxian Lu, Miaohang Shan* \\ Department of Intensive Care Unit, the First Affiliated Hospital of Jinan University, Guangzhou, China \\ Email address: \\ 105224073@qq.com (Miaohang Shan) \\ ${ }^{*}$ Corresponding author \\ To cite this article: \\ Xin Zhang, Wanxian Lu, Miaohang Shan. Predictive Value of Caprini Venous Thromboembolism Risk Assessment Model for Deep Vein \\ Thrombosis in Intensive Care Unit Non-surgical Patients. American Journal of Internal Medicine. Vol. 8, No. 1, 2020, pp. 40-44. \\ doi: $10.11648 /$ j.ajim.20200801.18
}

Received: February 9, 2020; Accepted: February 19, 2020; Published: February 28, 2020

\begin{abstract}
Objective: By evaluating the relationship between deep vein thrombosis (DVT) in intensive care unit (ICU) non-surgical patients and Caprini venous thromboembolism risk assessment model (Caprini model for short), the predictive value of Caprini model in ICU non-surgical patients was analyzed. Methods: 200 ICU non-surgical inpatients in the first affiliated hospital of Jinan university from April to September 2019 were retrospectively analyzed. General data of patients and the number of new DVT events were collected, and Caprini model was used for scoring the risk of venous thromboembolism (VTE). Results: There were 31 patients with DVT, accounting for $15.50 \%$, and 169 patients without new DVT (non-DVT). Caprini model score was $9.03 \pm 2.70$ in patients with DVT, higher than that in patients without DVT $(6.80 \pm 2.48, \mathrm{P}<0.001) .24$ $(12.00 \%)$ non-surgical ICU patients were at high risk of VTE and 171 cases $(85.50 \%)$ were at very high risk. Only one patient with DVT was at high risk of VTE (3.23\%), while the other 30 patients were at very high risk of VTE $(96.77 \%)$. There were 1 case in low risk of VTE (0.59\%), 4 cases in medium risk (2.37\%), 23 cases in high risk (13.61\%) and 141 cases in very high risk $(83.43 \%)$ in non-DVT group. There was no significant difference in VTE risk stratification between DVT patients and non-DVT patients $(\mathrm{P}=0.063)$. The receiver operating characteristic $(\mathrm{ROC})$ curve was plotted by using Caprini model score to predict DVT. The area under the ROC curve was 0.731 , and the $95 \%$ confidence interval was $0.663-0.791(\mathrm{P}<0.001)$. The optimal cut-off point was 7 , the sensitivity was $74.19 \%$, the specificity was $65.68 \%$ and Youden's index was 0.3897 . Conclusion: The incidence of high risk and very high risk of VTE in ICU non-surgical patients was high, and Caprini model could better predict the occurrence of DVT, so it was necessary to strengthen the nursing of ICU non-surgical patients and effectively prevent DVT.
\end{abstract}

Keywords: Intensive Care Unit, Deep Vein Thrombosis, Venous Thromboembolism, Risk Assessment Model, Prediction

\section{Introduction}

Venous thromboembolism (VTE) refers to the abnormal coagulation of blood in deep vein cavities, which blocks venous cavities and leads to venous returning disorders, mainly including deep vein thrombosis (DVT) and pulmonary thromboembolism (PE) [1]. In the United States, about 478,000 fatal VTE events occurred each year [2]. DVT often occurred in the lower limb vein. Studies had shown that $3 / 4$ of DVT occurred in non-surgical patients of internal medicine and only $25 \%$ patients who die of VTE in general hospitals had a recent surgical history, while the rest were non-surgical patients of internal medicine, accounting for $10 \%$ of the total deaths of internal medicine patients [3, 4]. Intensive care unit (ICU) patients were especially at high risk of DVT. Chinese studies reported that the incidence of DVT in ICU patients was from $9.7 \%$ to $27.0 \%$, while reports in Western countries were as high as from $24 \%$ to $40 \%$. Meanwhile, due to the hidden clinical manifestations of DVT, about $50 \%$ patients lacked typical clinical features of DVT, so the actual incidence of DVT might be higher [5-7]. It has been reported that about 50\% DVT patients would develop long-term sequelae of post-thrombus syndrome [5]. Therefore, early risk assessment and prevention were crucial to reduce the incidence of DVT in ICU patients 
and improve long-term prognosis [8, 9].

Early screening and evaluation of DVT were of great value in the prevention and treatment of VTE, especially fatal PE, so the prevention of VTE was more important than the treatment. Clinical studies have shown that the prediction of clinical probability in patients with suspected DVT had become an important basis for DVT diagnosis [10]. There were several risk assessment models for VTE, among which Caprini model was most commonly used in the non-surgical patients [2]. Caprini model had attracted more and more attention because it could be comprehensive scoring and rating based on common non-invasive related factors in clinical practice. Some studies have shown its effectiveness and reliability in the application of Caprini model to inpatients in internal and surgical departments, so it has been widely promoted in clinical work [11-13].

Therefore, this study was aim to analyze the predictive value of Caprini Model in ICU non-surgical patients' DVT by evaluating the relationship between DVT in ICU non-surgical patients and Caprini Model, so as to provide a clinical reference.

\section{Method}

\subsection{Study Population}

A total of 200 ICU non-surgical patients were collected from the first affiliated hospital of Jinan university from April to September 2019, with an average age of 61.85 years, including 135 males and 65 females. Basic diseases include: 50 cases of severe pneumonia $(25.00 \%), 11$ cases of multiple organ dysfunction syndrome (MODS, 5.50\%), 10 cases of acute coronary syndrome $(5.00 \%), 18$ cases of acute heart failure $(9.00 \%), 17$ cases of acute cerebral infarction $(8.50 \%)$, 7 cases of acute cerebral hemorrhage (3.5\%), 12 cases of acute gastrointestinal bleeding $(6.00 \%), 9$ cases of septic shock $(4.50 \%)$, and 66 cases of the others $(33.00 \%$, Figure 1$)$. Inclusion criteria: (1) age $\geq 18$ years; (2) length of stay: $3-30$ days; (3) admission to ICU without surgery; (4) informed consent and voluntary participation of the patient or family member. The exclusion criteria were: DVT occurred within 3 months before admission or lack of clinical data.

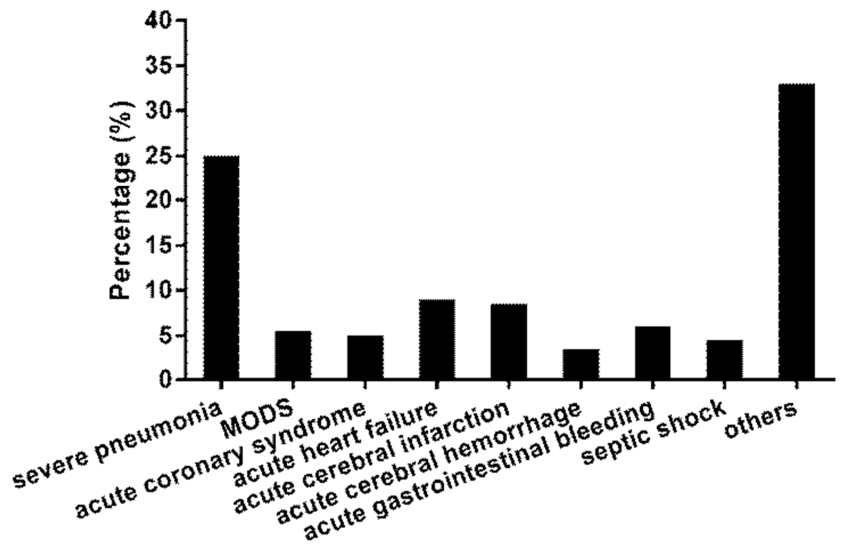

Figure 1. Distributions of basic diseases.

\subsection{Assessment Tools}

Using the 2010 Caprini model, 40 risk factors for venous thrombosis in all subjects were collected and assigned to each risk factor one by one. The items without data were denoted as "-". Different risk factors were assigned different values of 1-5. Caprini model scores were calculated, and the subjects were stratified into VTE risk: 0-1 was low risk, 2 was medium risk, 3-4 was high risk, and $\geq 5$ was very high risk [2].

\subsection{Evaluation Method}

Clinical data and laboratory examination results of all patients included in the study were collected, and the Caprini model was evaluated by professionally trained nurses within $8 \mathrm{~h}$ of admission, and risk stratification warnings were performed.

\subsection{DVT Assessment}

The formation of DVT of lower limbs was diagnosed by doppler ultrasound examination of deep vein of lower limbs conducted by two professional ultrasound doctors.

\subsection{Statistical Analysis}

Continuous variables are represented as mean \pm standard deviation (SD), while non-parametric variables are represented as median and quartile ranges. Categorical variables are expressed as frequency and percentage. Student's t-tests, analysis of variance (ANOVA), or nonparametric tests were used to compare continuous variables between groups. Differences between categorical variables were analyzed by chi-square tests or double-tailed Fisher exact tests, as applicable.

The accuracy and optimal threshold estimation of VTE predicted by Caprini model was analyzed by receiver operating characteristic (ROC) curve, and sensitivity, specificity, area under ROC Curve and Youden's index) were calculated.

All values are two-tailed, and $P<0.05$ was considered statistically significant. Data were analyzed using IBM SPSS Statistics version 25.0 for Windows (IBM, Armonk, NY, USA).

\section{Result}

\subsection{Clinical Characteristics}

There were 31 new cases of DVT, accounting for $15.50 \%$. Caprini score of ICU non-surgical patients was $7.15 \pm 2.64$, and Caprini score of DVT patients was $9.03 \pm 2.70$, higher than that of non-DVT patients $(6.80 \pm 2.48, \quad \mathrm{P}<0.001)$. Compared with non-DVT patients, DVT patients were older $(\mathrm{P}=0.009)$, and had a higher history of prior DVT $(\mathrm{P}=0.021)$. There were no significant differences in gender, diabetes, hypertension, coronary heart disease, previous cerebral infarction, smoking, drinking and mechanical ventilation history between DVT and non-DVT group (all $\mathrm{P}>0.05$, Table 1 and Figure 2). 
Table 1. Differences of demographic and clinical characteristics.

\begin{tabular}{|c|c|c|c|c|}
\hline & Non-DVT $(\mathrm{N}=169)$ & DVT $(\mathrm{N}=31)$ & Total $(\mathrm{N}=\mathbf{2 0 0})$ & $\mathbf{P}$ \\
\hline Age (years) & $60.38 \pm 18.93$ & $69.84 \pm 15.35$ & $61.85 \pm 16.70$ & 0.009 \\
\hline $41-60$ years $(\mathrm{N} / \%)$ & $44(26.04 \%)$ & $4(12.90 \%)$ & $48(24.00 \%)$ & 0.067 \\
\hline $61-74$ years $(\mathrm{N} / \%)$ & $52(30.77 \%)$ & $12(38.71 \%)$ & $64(32.00 \%)$ & \\
\hline$\geq 75$ years $(\mathrm{N} / \%)$ & $44(26.04 \%)$ & $13(41.94 \%)$ & $57(28.50 \%)$ & \\
\hline Gender & & & & 0.422 \\
\hline Male $(\mathrm{N} / \%)$ & $116(68.64 \%)$ & $19(61.29 \%)$ & $135(67.50 \%)$ & \\
\hline Female (N/\%) & $53(31.36 \%)$ & $12(38.71 \%)$ & $65(32.50 \%)$ & \\
\hline Diabetes (N/\%) & $40(23.67 \%)$ & $10(32.26 \%)$ & $50(25.00 \%)$ & 0.310 \\
\hline Hypertension (N/\%) & $77(45.56 \%)$ & $20(64.52 \%)$ & $97(48.50 \%)$ & 0.052 \\
\hline Coronary heart disease $(\mathrm{N} / \%)$ & $19(11.24 \%)$ & $5(16.13 \%)$ & $24(12.00 \%)$ & 0.442 \\
\hline Previous cerebral infarction (N/\%) & $26(15.38 \%)$ & $7(22.58 \%)$ & $33(16.50 \%)$ & 0.321 \\
\hline History of prior DVT (N/\%) & $2(1.18 \%)$ & $3(9.68 \%)$ & $5(2.50 \%)$ & 0.021 \\
\hline Smoking (N/\%) & $78(46.15 \%)$ & $16(51.61 \%)$ & $94(47.00 \%)$ & 0.576 \\
\hline Drinking (N/\%) & $12(7.10 \%)$ & $4(12.90 \%)$ & $16(8.00 \%)$ & 0.463 \\
\hline Mechanical ventilation history (N/\%) & $6(3.55 \%)$ & $2(6.45 \%)$ & $8(4.00 \%)$ & 0.795 \\
\hline Caprini model score & $6.80 \pm 2.48$ & $9.03 \pm 2.70$ & $7.15 \pm 2.64$ & $<0.001$ \\
\hline
\end{tabular}

DVT: deep vein thrombosis.

Age and Caprini model score were expressed as mean and standard deviation.

$\mathrm{P}$ value for analysis of comparison between non-DVT patients and DVT patients.

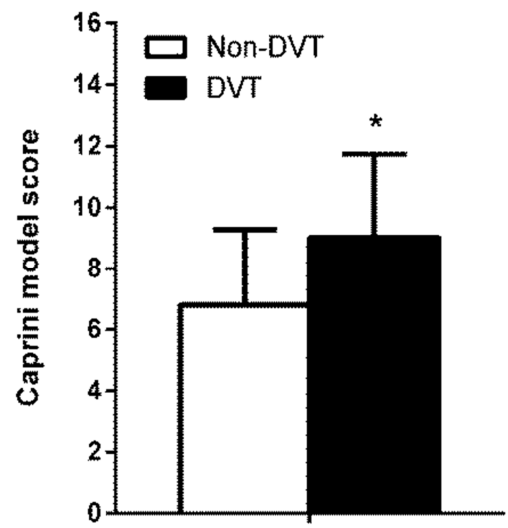

(* indicated comparison with non-DVT and DVT group, $\mathrm{p}<0.05$ ).

Figure 2. Caprini model score between non-DVT patients and DVT patients.

\subsection{Caprini Model Score and VTE Risk Stratification}

According to Caprini model score, risk stratification of VTE was conducted. 24 cases $(12.00 \%)$ of ICU non-surgical patients were at high risk of VTE, and 171 cases were at very high risk, up to $85.50 \%$. Among the DVT patients, only one patient was at high risk of VTE (3.23\%) and the other 30 patients were very high risk of VTE $(96.77 \%)$. Non-DVT patients had 1 low risk $(0.59 \%), 4$ medium risk $(2.37 \%), 23$ high risk $(13.61 \%)$ and 141 very high risk $(83.43 \%)$ cases of VTE. There was no significant difference in risk stratification of VTE between DVT and non-DVT patients $(\mathrm{P}=0.063$, Table 2 and Figure 3).

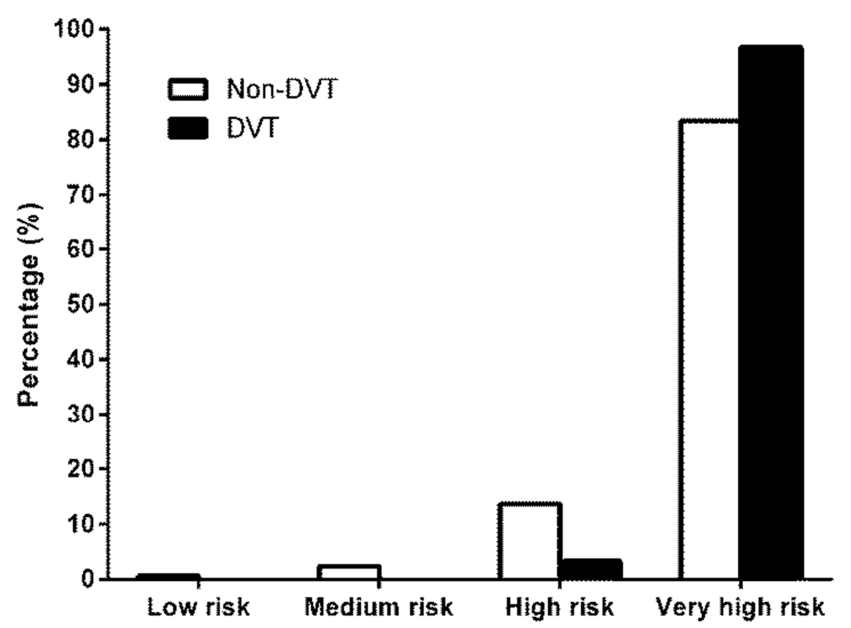

Figure 3. VTE risk stratification between non-DVT and DVT patients.

Table 2. VTE risk stratification between non-DVT and DVT patients.

\begin{tabular}{llll}
\hline & Non-DVT (N=169) & DVT (N=31) & Total (N=200) \\
\hline low risk (N/\%) & $1(0.59 \%)$ & 0 & $1(0.50 \%)$ \\
medium risk (N/\%) & $4(2.37 \%)$ & 0 & $4(2.00 \%)$ \\
high risk (N/\%) & $23(13.61 \%)$ & $1(3.23 \%)$ & $24(12.00 \%)$ \\
very high risk (N/\%) & $141(83.43 \%)$ & $30(96.77 \%)$ & $171(85.50 \%)$ \\
\hline
\end{tabular}

DVT: deep vein thrombosis.

\subsection{ROC Curve}

The ROC curve was plotted by using Caprini model score to predict DVT. The area under the ROC curve was 0.731 , and the $95 \%$ confidence interval was $0.663-0.791(\mathrm{P}<0.001)$. The optimal cut-off point was 7 , the sensitivity was $74.19 \%$, the specificity was $65.68 \%$ and Youden's index was 0.3897 (Table 3 and Figure 4). 
Table 3. ROC curve.

\begin{tabular}{|c|c|c|c|c|c|c|}
\hline $\begin{array}{l}\text { Area under the ROC } \\
\text { curve }\end{array}$ & $\begin{array}{l}95 \% \text { CI of area under the } \\
\text { ROC curve }\end{array}$ & $\mathbf{P}$ & $\begin{array}{l}\text { optimal cut-off } \\
\text { point }\end{array}$ & Sensitivity (\%) & Specificity (\%) & Youden's index \\
\hline 0.731 & $0.663-0.791$ & $<0.001$ & 7 & 74.19 & 65.68 & 0.3987 \\
\hline
\end{tabular}

CI: confidence interval; ROC: receiver operating characteristic.

$\mathrm{P}$ value for area under the ROC curve.

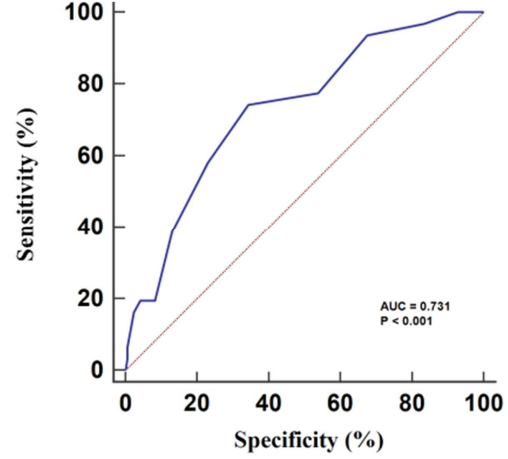

Figure 4. ROC curve.

\section{Discussion}

Early diagnosis was crucial to DVT, especially in the ICU patients, so a rapid and effective non-invasive detection method was needed in clinical practice. This study showed that 31 patients among 200 ICU non-surgical patients had new DVT, accounting for $15.50 \%$. Caprini model score was $9.03 \pm 2.70$ in patients with DVT, higher than that in patients without DVT (6.80 $\pm 2.48, \mathrm{P}<0.001) .24$ (12.00\%) non-surgical ICU patients were at high risk of VTE and 171 cases $(85.50 \%)$ were at very high risk. Only one patient with DVT was at high risk of VTE $(3.23 \%)$, while the other 30 patients were at very high risk of VTE $(96.77 \%)$. There were 1 case in low risk of VTE $(0.59 \%), 4$ cases in medium risk $(2.37 \%), 23$ cases in high risk (13.61\%) and 141 cases in very high risk $(83.43 \%)$ in non-DVT group. There was no significant difference in VTE risk stratification between DVT patients and non-DVT patients $(\mathrm{P}=0.063)$. The ROC curve was plotted by using Caprini model score to predict DVT. The area under the ROC curve was 0.731 , and the $95 \%$ confidence interval was 0.663-0.791 $(\mathrm{P}<0.001)$. The optimal cut-off point was 7 , the sensitivity was $74.19 \%$, the specificity was $65.68 \%$ and Youden's index was 0.3897 .

The body of ICU patients was in a state of organ failure and acute stress, therefore, the procoagulant system in vivo was abnormal and their body were often in a state of braking, which was likely to lead to the formation of systemic microthrombosis, thus, DVT of lower limbs became more common [3]. Nowadays, DVT has become a worldwide public health care problem due to its high morbidity and mortality. Venography was the gold standard of DVT diagnosis, while color doppler ultrasound was a simple and fast method to screen DVT [14]. At present, more and more research focuses on early screening and prevention of DVT. According to some studies, DVT was considered to be "the single most likely cause of death to be prevented" [15]. The guidelines for the diagnosis and treatment of thrombus recommend the use of drugs and mechanical prevention methods for high-risk patients with DVT [3, 10]. Therefore, it was particularly important to use effective, simple, convenient and economic prediction and evaluation tools to screen high-risk groups for venous thrombosis, and to take targeted preventive measures according to the risk level.

In recent years, clinical researchers have developed assessment tools to predict the likelihood of VTE in order to facilitate timely diagnosis and reduce unnecessary testing and financial burden. Caprini model has attracted more and more attention among VTE risk assessment tools. Caprini model was first reported in 1980 and updated regularly, and relatively mature in 2010, which was the most widely used model [2]. Because of its comprehensive content, simple and convenient assessment and strong feasibility, Caprini model has been translated into 12 languages and applied in clinical practice in the United States, Japan, South Korea, China and other countries at present [16]. According to clinical and laboratory data, 40 risk factors were assigned a score of 1-5, and the risk of thrombosis was stratified according to the total score to predict the probability of VTE in patients: the low risk layer was $2 \%$, the middle risk layer was $10 \%-20 \%$, the high risk layer was $20 \%-40 \%$, and the very high risk layer was $40 \%-80 \%$ [11]. Bahl V et al. used Caprini model to verify 8,216 inpatients in general surgery, vascular surgery and urology department, and the results showed that Caprini model had the best predictability when the critical value was 5 [11]. In this study, the optimal threshold value was 7 , with a sensitivity of $74.19 \%$ and a specificity of $65.68 \%$, suggesting that different races, basic disease, treatment schemes and living habits might affect the optimal threshold value.

According to risk stratification, Caprini model recommended corresponding preventive measures, including the type and duration of preventive measures. Low-risk patients did not need special treatment and should had early activity, including active or passive movement. In the middle and high risk patients, the implementation of basic prevention should be early activities combined with physical prevention or drug prevention. Physical prophylaxis at present mainly included intermittent pressure inflator pump and antithrombotic stretch socks. Drug prophylaxis was considered when anticoagulant contraindications were excluded and anticoagulants were administered to patients. Very high risk patients were given physical and drug prophylaxis on the basis of basic prophylaxis. Early risk screening for patients was not only conducive to timely detection of DVT risk in patients, but also could make nursing prevention more targeted and reduce the occurrence of DVT at the source [17]. 
This study had some advantages and limitations. First of all, we evaluated the diagnostic value of Caprini model in the diagnosis of DVT. Secondly, Caprini model, as a non-invasive and efficient index, had a high predictive value for DVT and was suitable for clinical application. Limitations included the following points: the sample size was relatively small; due to only one center was included, multicenter data and follow-up studies are necessary; and lack of some biochemical indexes involved in Caprini model (such as anticardiolipin antibody, lupus anticoagulant, prothrombin 20210 and leiden factor).

\section{Conclusion}

DVT is an important complication affecting recovery of ICU patients. This study shows that Caprini model can better predict DVT in ICU non-surgical patients, which is worthy of being popularized in clinical work. According to DVT risk stratification, the nursing of non-surgical patients in ICU should be strengthened to effectively prevent DVT and improve survival rate and quality of life of patients.

\section{Competing Interests}

The authors declare that they have no competing interests.

\section{Acknowledgements}

The authors would like to acknowledge all patients and their families for participating in this study.

\section{References}

[1] SchItIman D, Ageno W, KonstanIinides SV. Venous hromboebolism: past, present and future [J]. Thromb Haemost, 2017, 117 (7): 1219-1229.

[2] Caprini J A. Risk assessment as a guide to thrombosis prophylaxis [J]. Curr Opin Pulm Med, 2010, 16 (5): 448-452.

[3] Sevens S M, Douketis J D. Deep vein thrombosis prophylaxis in hospitalized medical patients: current recommendations, general rates of implementation, and initiatives for improvement [J]. Clin Chest Med, 2010, 31 (4): 675-689.

[4] Hostlerd C, Marxe S, Mooresl K, et al. Validation of the international medical prevention registry on venous thromboembolism bleeding risk score [J]. Chest, 2016, 149 (2): 372-379.
[5] ObIa T, Pannuccl C J, Nackashl A, et al. Validation of the Caprini venous thromboembolism risk assessment model in critically ill surgical patients [J]. JAMA Surg, 2015, 150 (10): 941-948.

[6] Miner C, Potton L, Bonadona A, et al. Venous thromboembolism in the ICU: main characteristics, diagnosis and thromboprophyIaxis [J]. Crit Care, 2015, 19: 287.

[7] Geerts W H, Heit J A, Clagett G P, et al. Prevention of venous thromboembolism [J]. Chest, 2001, 119 (1 Suppl): 132S-175S.

[8] Cullough M, Kholdani C, Zamanian RT. Prevention of deep vein thrombosis and pulmonary embolism in high-risk medical patients [J]. Clin Chest Med. 2018, 39 (3): 483-492.

[9] Brien A, Redley B, Wood B, et aI. STOPDVTs: Development and testing of a clinical assessment tool to guide nursing assessment of postoperative patients for deep vein thrombosis [J]. J Clin Nurs, 2018, 27 (9): 1803-1811.

[10] Qaseem A, Snow V, Barry P, et al. Current diagnosis of venous thromboembolism in primary care: A clinical practice guideline from the American academy of family physicians and the American college of physicians [J]. Ann Intern Med. 2007.146 (6): 454-458.

[11] Bahl V, Hu HM, Henke PK, et al. A validation study of a retrospective venous thromboembolism risk scoring method [J]. Ann Surg, 2010, 251 (2): 344-350.

[12] Liu X, Liu C, Chen X, et al. Comparison between Caprini and Padua risk assessment models for hospitalized medical patients at risk for venous thromboembolism: a retrospective study [J]. Interact Cardiovasc Thorac Surg, 2016, 23 (4): 538-543.

[13] Zhou Hx, Peng LQ, Yan Y, et al. Validation of the Caprini risk assessment model in Chinese hospitalized patients with venous thmmboembolism [J]. Thromb Res, 2012, 130 (5): 735-740.

[14] SIone J, Hangge P, AIbadawi H, et al. Deep vein thrombosis: pathogenesis, diagnosis, and medical management [J]. Cardiovasc Diagn Ther, 2017, 7 (3): 276-284.

[15] Zhang H, Mao P, Wang C, eI al. Incidence and risk factor of deep vein thrombosis (DVT) after total hip or knee arthroplasty: a retrospective study with routinely applied venography [J]. Blood Coagul Fibrinolysis, 2017, 28 (2): 126-133.

[16] Tongiputn S, Kunanusont N, Senjuntichai C, et al. Lower extremity deep venous thrombosis among that patients with stroke [J]. Neurol J Southeast Asia, 1999, 4: 13-18.

[17] Alper EC, Ip Ik, Balthazar P, et al. Risk stratification model: lower extremity ultrosonography for hospitalized patients with suspected deep vein thrombosis [J]. J Gen Intern Med, 2018, 33 (1): 21-25. 